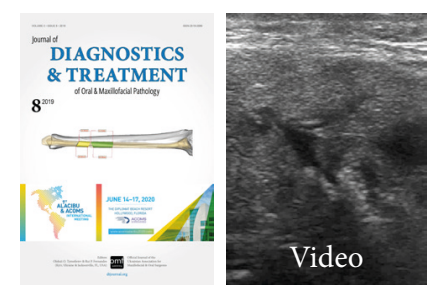

Pics in Oral \& Maxillofacial Surgery + Video

Camilo Mosquera, Editor

\title{
| Ultrasound in the Detection of Floating Sialoliths
}

Oleksii 0. Tymofieiev \& Olha S. Cherniak
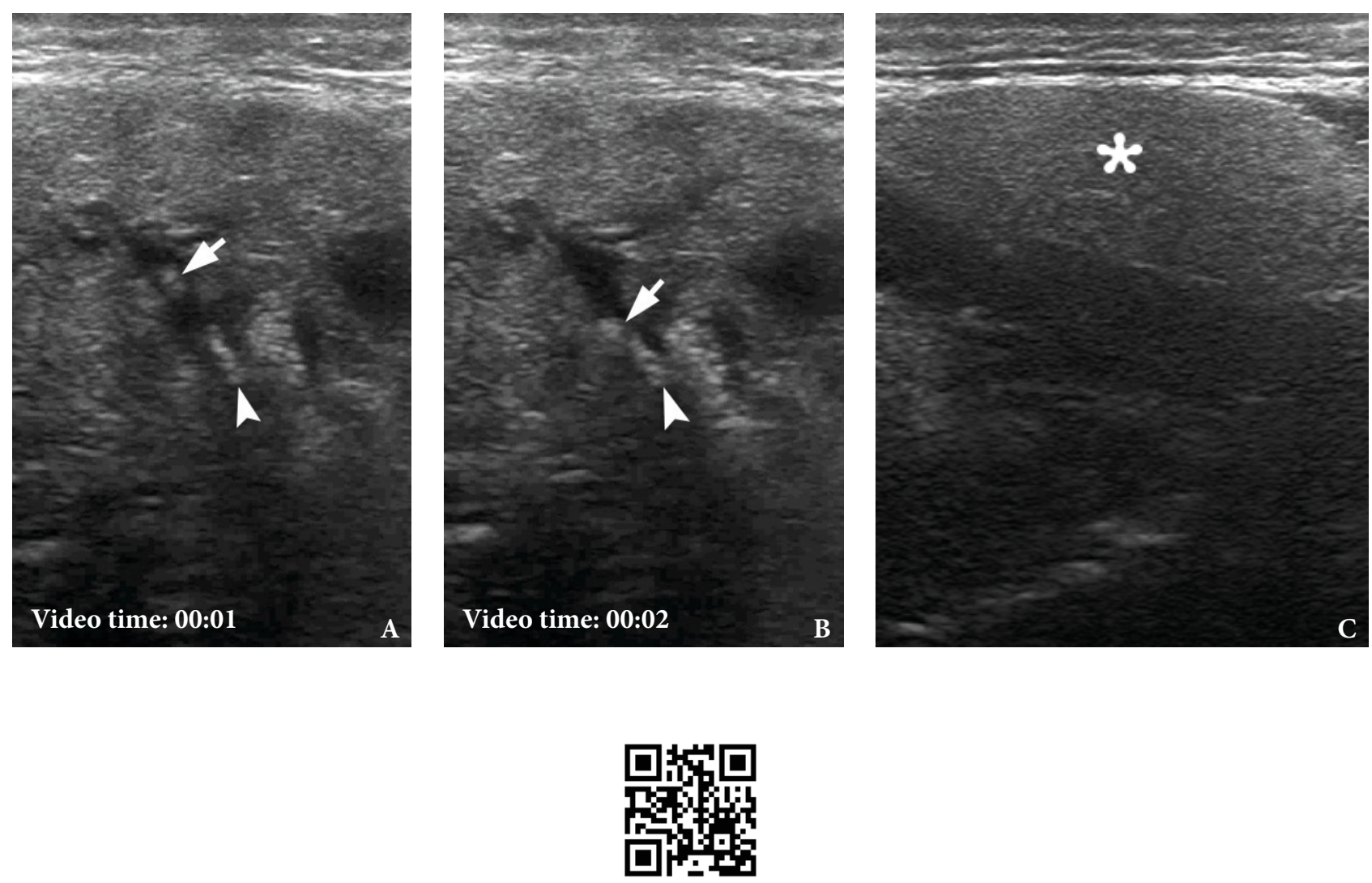

QR code leads to that video at

DTJournal's YouTube channel

Videos DTJournal

a ScD, Professor; Head, Maxillofacial Surgery Department, NMAPE, Kyiv, Ukraine.

http://dx.doi.org/10.23999/j.dtomp.2019.8.2.

(C) 2019 OMF Publishing, LLC. This is an open access article under the CC

tymofeev@gmail.com (Oleksii Tymofieiev)

BY license (http://creativecommons.org/licenses/by-nc/4.0/)

${ }^{b}$ Head, Department of Ultrasound, Regional Diagnostic Center, Kyiv

Regional Clinical Hospital, Kyiv, Ukraine.

cherniak.os@gmail.com (Olha Cherniak)

dtjournal.org 


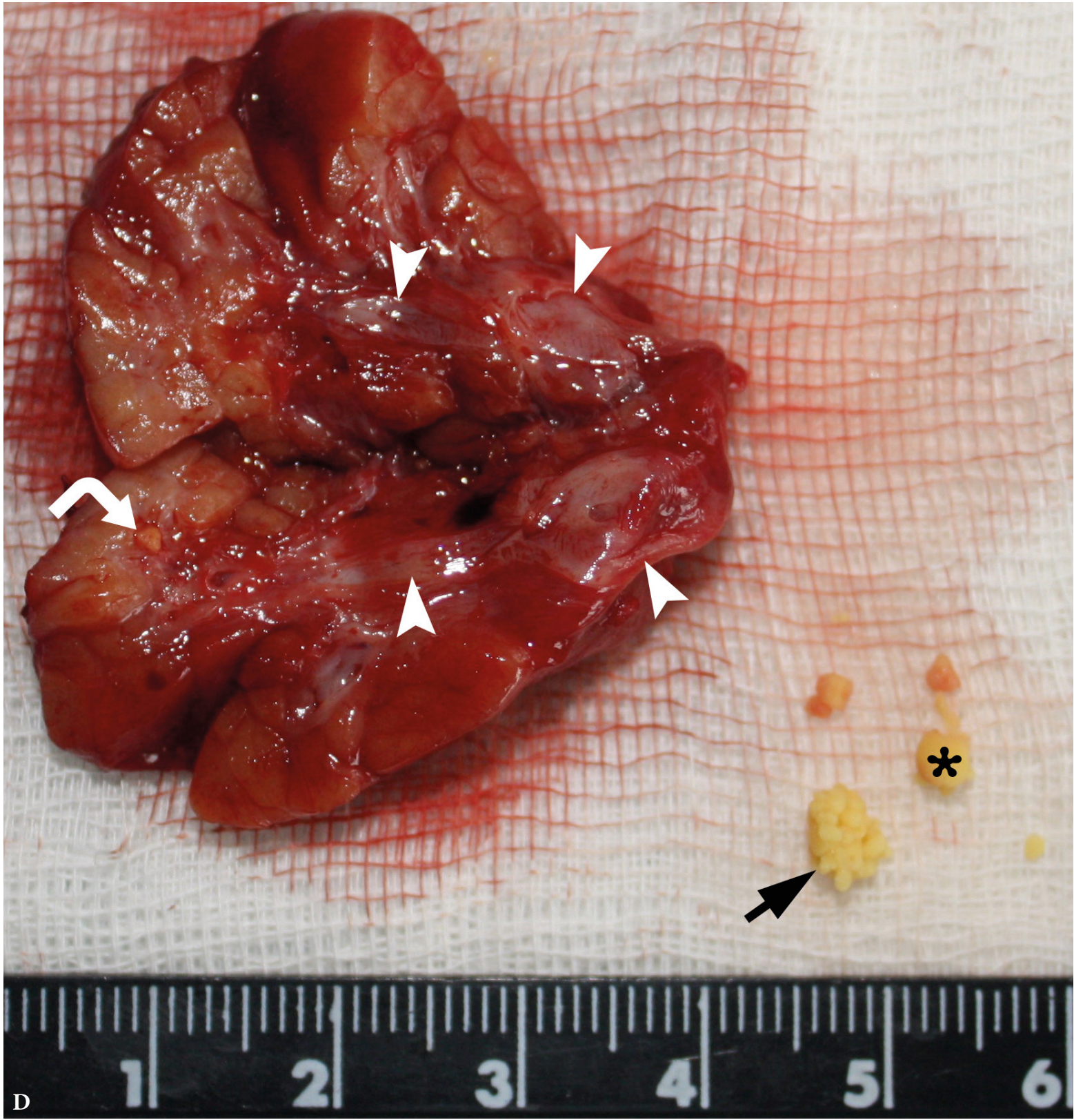

A 36-year-old man with a 3-year history of recurrent salivary colic was referred to a maxillofacial surgery department. Gray scale ultrasound (US) showed enlarged right submandibular gland, significantly dilated intraglandular duct with two sialoliths (with an artifact of acoustic shadowing) inside, one - floating (Video-Panel A and B, arrow) and another - nonmovable (arrowhead). Left nonsymptomatic normal in size gland (asterisk) is showed at Panel C. The affected gland was excised under general anesthesia due to the diagnosis of chronic submandibular obstructive sialolithiasis. Intraglandular duct contained two yellowish stones, first was an oval form with a pellet surface (Panel D, arrow), second - a round shaped with a smooth surface (Panel $\mathrm{D}$, asterisk) and it was presented at US as a floating sialolith; both are easily crumbled on palpation. As the specimen and intraglandular duct were dissected longitudinally, that's why dissected intraglandular duct (Panel D, arrowheads) is visible in both parts of the gland. Also, a 1 small calculus (Panel D, curved arrow) was found in the parenchymal ducts. Postoperative period was smooth, and 1-year follow-up after surgery, the patient has no complaints. = DTJournal 